\title{
Zahnfrakturen beim Pferd und deren Therapiemöglichkeiten
}

\author{
Anna Altersberger
}

Zahnfrakturen bei Pferden werden als Zufallsbefunde im Rahmen von Routinezahnbehandlungen entdeckt. Allerdings müssen sie bei Auftreten akuter klinischer Symptome zu den zahnmedizinischen Notfällen gezählt werden. Welche Formen von Zahnfrakturen es gibt und nach welchen Kriterien bestehende Therapiemöglichkeiten ausgewählt werden, soll der folgende Artikel vermitteln. Er gibt einen Überblick über häufig auftretende Zahnfrakturen sowie über Instrumente, Materialien und Arbeitsschritte, die zur Versorgung notwendig sind.

\section{Einführung}

Zahnfissuren und -frakturen können grundsätzlich an allen Zahntypen des Pferdes auftreten. Sie gehören für Tierärzte, die auf Zahnheilkunde spezialisiert sind, zum Arbeitsalltag. Nicht zu unterschätzen ist der Anteil an Zahnfrakturen, der bei Routinezahnbehandlungen festgestellt wird. Akute Frakturen dagegen sind nicht selten Grund für einen Anruf im Notdienst, da bei betroffenen Pferden plötzlich vermehrter Speichelfluss, Maulgeruch und Kauprobleme - wie etwa das Wickelkauen - beobachtet werden können $[9,11]$.

Man unterscheidet traumatisch bedingte, iatrogene und idiopathische Zahnfrakturen [11].

\section{Zahnfrakturen der einzelnen Zahntypen}

Iatrogene Zahnfrakturen können in vielen Fällen vermieden werden, indem vor dem Einsetzen eines Maulgatters stets die Schneidezähne auf Stabilität überprüft werden und auf den Einsatz von Maulkeilen und Zahncuttern verzichtet wird [11]!

\section{Schneidezähne}

An den Schneidezähnen sind häufig traumatisch bedingte Zahnfrakturen zu beobachten, da diese Zähne sehr exponiert liegen. Weichteilgewebe und Kieferanteile können hierbei mitbetroffen sein. Verletzungsursachen sind u.a. Tritte, Hängenbleiben beim Spielen oder Kauen auf Gegenständen, Wetzen oder Koppen, aber auch Stürze und Kollisionen, z.B. im Zusammenhang mit schwerer Kolik oder Aufstehversuchen nach einer Allgemeinanästhesie [1,9,11].

Traumatisch bedingte Schneidezahnfrakturen treten vermehrt bei jungen Pferden auf [1]. Sind mehrere Schneidezähne betroffen, ist regelmäßig der Alveolarknochen mitbeteiligt (Zahnfachfraktur), die Zähne sind nach labial oder lingual verlagert und die Frakturlinie kann weit apikal im Wurzelbereich der Zähne liegen. Hier ist zur Frakturversorgung eine Drahtzerklage das Mittel der Wahl.

Die sog. „3er“ (Eckschneidezähne) sind aufgrund ihrer Lage und der Druckverhältnisse anfälliger für Traumata als die übrigen Schneidezähne. Zudem treten an den „3ern“ besonders bei älteren Pferden (> 15 Jahre) idiopathische Zahnfrakturen auf. Diese Zähne können durch Karies, Parodontitis oder EOTRH (Equine Odontoclastic Tooth Resorption and Hypercementosis) vorgeschädigt sein [11].

Um iatrogene Schneidezahnfrakturen zu vermeiden, empfiehlt sich vor Verwendung eines Maulgatters immer eine Adspektion und Palpation der Schneidezähne. Hierbei können überstehende oder instabile Zähne sowie ein unregelmäßiger Schneidezahnbogen erkannt werden. Gegebenenfalls muss dieser vor Einsetzen des Maulgatters beispielsweise mit einer Trennscheibe angeglichen oder begradigt werden.
Die Frakturlinien an den Schneidezähnen verlaufen oft transversal auf oder unter Zahnfleischniveau [3,11].

\section{Hengstzähne}

Auch Hengstzähne sind aufgrund ihrer Lage in der Lade anfällig für Zahnfrakturen - z. B. durch Hängenbleiben in Gittern oder Stricken. Diese Frakturen gehen oft mit Pulpabeteiligung einher und zeigen einen meist schräg bis unterhalb des Zahnfleischniveaus reichenden Verlauf.

Die massive Anlagerung von Zahnstein und in der Folge die Entstehung von Parodontitis sowie die Beteiligung von Karies oder EOTRH begünstigen Frakturen der Hengstzähne [11].

\section{Backenzähne}

Die Backenzähne frakturieren seltener traumatisch als die anderen Zahntypen, da die Zähne durch Wange und Muskulatur besser geschützt sind [11]. Vorwiegend treten idiopathische Backenzahnfrakturen auf. Die Oberkieferbackenzähne, besonders die Molaren 109 und 110 bzw. 209 und 210, sind häufiger betroffen als die Unterkieferbackenzähne [8] und in der Mehrzahl der Fälle ist der Frakturverlauf sagittal [3].

Es existiert zur Unterscheidung der Backenzahnfrakturen folgende einfache Einteilung:

- Sagittalfrakturen mit Beteiligung der Pulpenpositionen (Slabfrakturen),

- Sagittalfrakturen durch die Mittellinie bzw. Infundibula des Zahnes sowie

- sonstige Frakturformen $[2,3,8]$.

Aufgrund sehr vielfältiger Frakturformen an den Backenzähnen bietet sich für eine bessere Orientierung am Zahn und eine korrekte Dokumentation eine genauere Unterteilung nach folgenden Kriterien an $(\triangleright$ Abb. 1): 


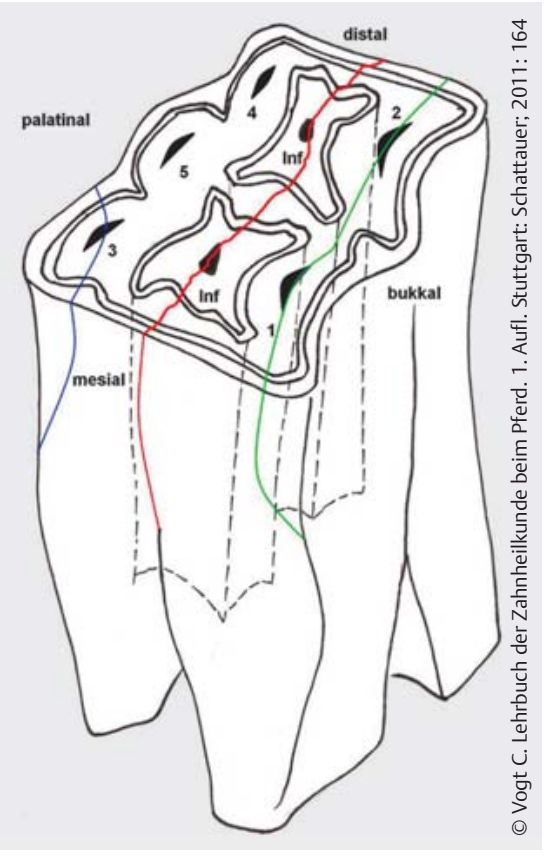

Abb. 1 Schema der Backenzahnfrakturen. Sagittalfraktur durch Pulpenpositionen 1 und 2 (grün), Sagittalfraktur durch die Infundibula (rot), Kronenteilfraktur palatinomesial (blau).

- Frakturebene (sagittal, transversal, horizontal)

- Lokalisation auf der Okklusionsfläche (bei Kronenteilfrakturen z.B. bukkodistal, palatinomesial)

- Beteiligung von Strukturen auf der Okklusionsfläche (Pulpenpositionen/Infundibula) und

- Öffnung der Pulpa
Sollten Pulpenpositionen bei einer

Zahnfraktur miteinbezogen sein, bedeutet dies nicht immer eine Öffnung der Pulpa $[10,11]$.

Sagittalfrakturen durch die Infundibula der Oberkieferbackenzähne treten nach Vorschädigung durch kariöse Prozesse auf, die zum Substanzverlust aufgrund von Entmineralisierung der Zähne führen. Sie können bis in den Apikalbereich des Zahnes reichen. Betroffen sind meist die „9er“ [11].

Laut histologischen Untersuchungen sind die Frakturbereiche von Sagittalfrakturen mit Beteiligung der Pulpenpositionen (Slabfrakturen) anatomische Schwachstellen, da hier die mineralisierten Zahngewebe am dünnsten sind. Sie zeigen häufig einen Frakturverlauf durch die beiden lateralen Pulpenpositionen. Es gibt nur selten Anzeichen einer Vorschädigung der Pulpa $[2,3,5]$.

Bei der Verwendung von Maulkeilen oder Zahncuttern können Backenzähne iatrogen frakturieren, weshalb auf den Einsatz solcher Instrumente unbedingt verzichtet werden sollte. Große Sorgfalt sollte stets bei der Extraktion und Repulsion von Backenzähnen angewandt werden, um Nachbarzähne nicht zu schädigen [11].
Die Festigkeit der Zähne präseniler und seniler Gebisse geht im Laufe der Zeit aufgrund altersbedingter Veränderungen, denen die Zahnhartsubstanzen und der Zahnhalteapparat unterliegen, verloren. Bei diesen Pferden kann bereits normaler Kaudruck zu Fissur und Fraktur von Zähnen führen [6].

\section{Klinische Symptome}

Die klinische Symptomatik stellt sich bei Zahnfrakturen sehr unterschiedlich dar. Oft sind die Pferde symptomfrei und Zahnfrakturen werden nur im Rahmen der jährlichen Zahnkontrolle festgestellt $[5,8]$. Bei akuten Zahnfrakturen ist es möglich, dass die Pferde vermehrt, gegebenenfalls blutig speicheln, Wickel kauen, Maulgeruch haben oder dass sie ihr Futter verweigern. Ferner kann es zu Rittigkeitsbeschwerden, Verhaltensproblemen oder Gewichtsverlust kommen [4,5, 8,11]. Bei Schneidezahnverletzungen können Blutungen und Schwellungen der Weichteilgewebe oder Kopfschütteln beobachtet werden. Das Allgemeinbefinden ist in der Regel nahezu ungestört und wird die Verletzung nicht zeitig bemerkt, setzen die Pferde schnell wieder ihre gewohnte Futteraufnahme fort. Bei älteren Frakturen wird unter Umständen ausschließlich unangenehmer Maulgeruch wahrgenommen [9].

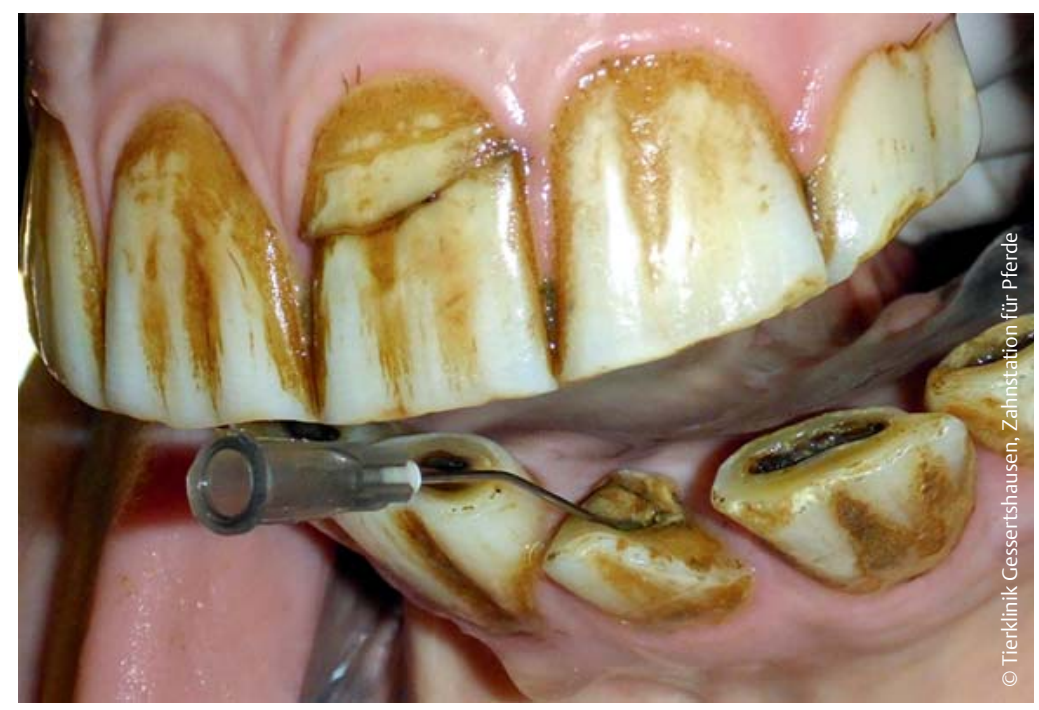

Abb. 2 Zahnfrakturen von 201 und 301. Sondieren der Pulpa von 301 mit einer Kanüle, ein Teil der klinischen Krone fehlt bereits; gut erkennbare Frakturlinie des Zahnes 201.

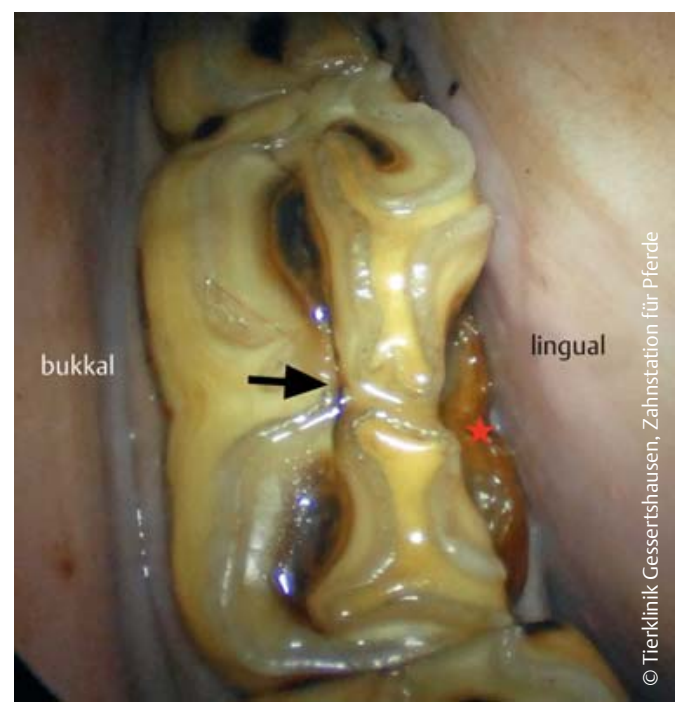

Abb. 3 Endoskopische Aufnahme eines Unterkieferbackenzahnes: linguomesiale Kronenteilfraktur (Stern) und Fissur zwischen den bukkalen Pulpenpositionen (Pfeil). 


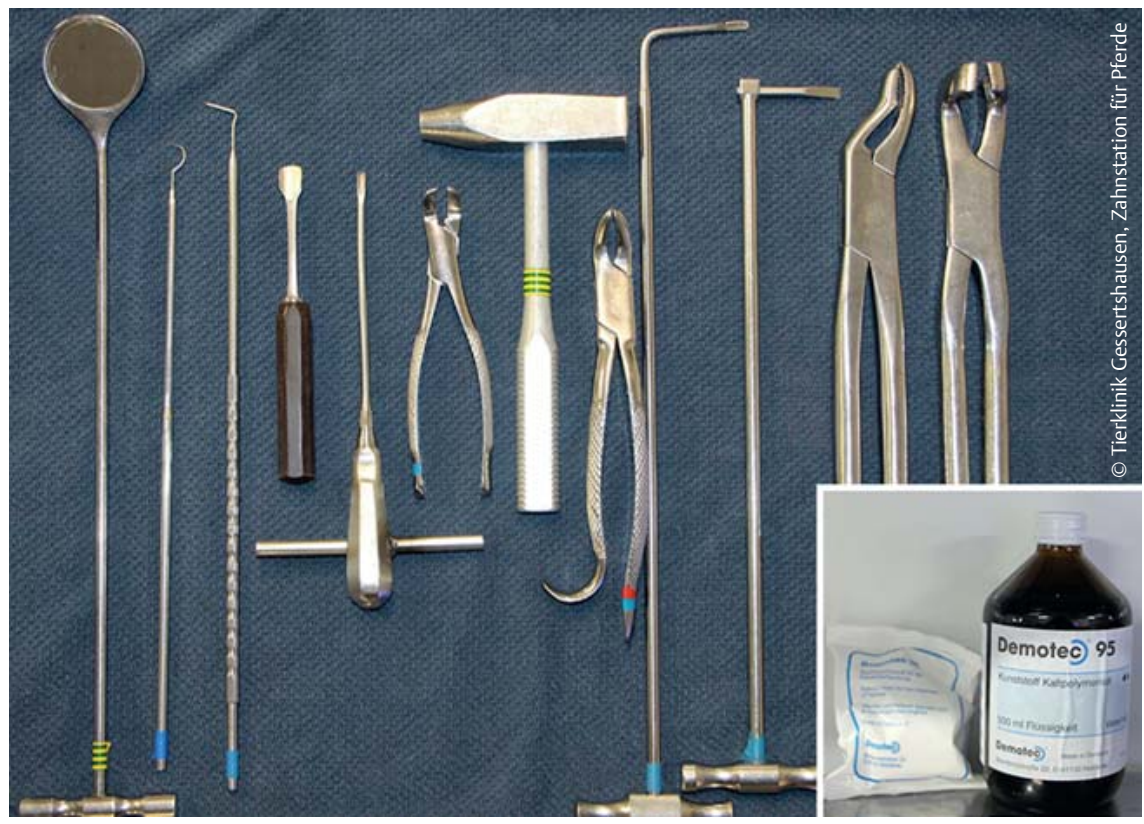

Abb. 4 Instrumente v.I.n. r.: Zahnspiegel, Sonden, Elevatoren/Hebel für Schneidezähne, Interdentalspreizer, Hammer, kleine Zange, Elevatoren/Picks für Backenzähne, Extraktionszangen, Füllmaterial (Demotec ${ }^{\circledR}$ ).

In manchen Fällen wird eine Umfangsvermehrung der Backe durch Futteranschoppung im Bereich der Zahnfraktur bemerkt $[5,11]$. Durch Fragmente, die nach bukkal oder lingual/palatinal verlagert sind, entstehen Schleimhautulzerationen [5,8]. Im Falle einer Apikalinfektion des betroffenen Zahnes können Knochenauftreibungen, Fisteln oder Nasenausfluss auftreten [4, 11]. Bei älteren Zahnfrakturen wird der Gegenspieler überlang, da er ungenügend abgenutzt wird [6].

\section{Diagnostik}

Die Erhebung des Vorberichts und eine Allgemeinuntersuchung erfolgen vor der speziellen Untersuchung der Maulhöhle und der umgebenden Strukturen [1]. Einige Zahnfrakturen sind offensichtlich, beispielsweise im Bereich der Schneidezähne, da Anteile der klinischen Krone oft mobil oder verlagert sind und bereits fehlen können ( $\mathbf{A} \mathbf{b} \mathbf{b} . \mathbf{2}$ ). Diese Frakturen sind leicht zu erkennen [6].

Es empfiehlt sich grundsätzlich, eine sorgfältige Untersuchung der gesamten Maulhöhle unter Sedierung durchzuführen. Die Palpation der einzelnen Zähne zur Überprüfung der Mobilität und Vollständigkeit ist ratsam. Besteht der Verdacht einer Zahnfachfraktur oder einer Kieferfraktur bzw. -fissur, sollten vor dem Einsetzen eines Maulgatters Röntgenaufnahmen angefertigt werden. Es existiert die Gefahr eines zusätzlichen iatrogenen Traumas durch Verwendung eines Maulgatters bei Vorliegen einer Knochenfraktur. Die Adspektion der Maulhöhle erfolgt bei eingesetztem Maulgatter mit guter Lichtquelle (Kopflampe) und Zahnspiegel oder starrem Endoskop, da gerade im Backenzahnbereich Frakturen und Fissuren übersehen werden können ( Abb. 3) [6]. Die Endoskopie stellt außerdem ein nützliches Instrument zur Dokumentation dar, die nicht nur bei Verlaufskontrollen von Bedeutung ist.

Sonden werden zur Untersuchung und Kontrolle auf das Vorliegen eröffneter Pulpenhöhlen genutzt [9]. An den Schneidezähnen können hierzu auch Kanülen verwendet werden ( $\bullet$ Abb. 2).

Röntgenaufnahmen in mehreren Ebenen geben gerade im Schneidezahnbereich Aufschluss über die Lage, Anzahl und den Verlauf der Frakturlinien [9]. Darüber hinaus sind sie hinweisend bei Verdacht auf eine Apikalinfektion [11] und eignen sich auch bei den Backenzähnen für die Beurteilung hinsichtlich sinnvoller Therapiemaßnahmen.

\section{Kasten 1: Instrumentarium}

Maulgatter

Dentalhalfter

Lichtquelle (Kopflampe)

Zahnspiegel

Sonden

Endoskop

Röntgen

Elevatoren, Hebel und Picks

Interdentalspreizer für Schneidezähne

Hammer

Extraktionszangen

Schleifmaschinen

Spezialausrüstung für Endodontie und

Backenzahnextraktion

Füllmasse z. B. Kunstharz (Demotec ${ }^{\circledR}$ )

Eine lückenlose Dokumentation der Zahnfrakturen (Zahnbefundbogen, Fotos) erleichtert die Beurteilung des Verlaufs im Rahmen regelmäßig stattfindender Nachkontrollen.

\section{Therapiemöglichkeiten häufig auftretender Zahnfrakturen}

Bedeutend ist bei der Therapieauswahl und der Beurteilung der Prognose für den Erhalt des frakturierten Zahnes die Beteiligung der Pulpa!

\section{Vorbereitung und Instrumentarium}

Die Therapiemaßnahmen können in der Regel unter Sedierung (häufige Kombination: Detomidinhydrochlorid [0,01$0,04 \mathrm{mg} / \mathrm{kg}$ i.v.] und Butorphanoltartrat [0,015 mg/kg i.v.]) durchgeführt werden. Bei der Entfernung von Zahnfragmenten, bei Zahnextraktionen und endodontischen Maßnahmen sollte eine Lokal- oder Leitungsanästhesie in Betracht gezogen werden. Zusätzlich wird die parenterale Gabe eines nichtsteroidalen Antiphlogistikums, z.B. Flunixin-Meglumin $(1,1 \mathrm{mg} / \mathrm{kg}$ i.v.) empfohlen. Außerdem muss stets auf eine ausreichende Tetanusprophylaxe geachtet werden [1]!

In $>$ Abb. 4 und $>$ Kasten 1 sind wichtige Instrumente und Materialien für die Diagnostik und Behandlung aufgeführt. 


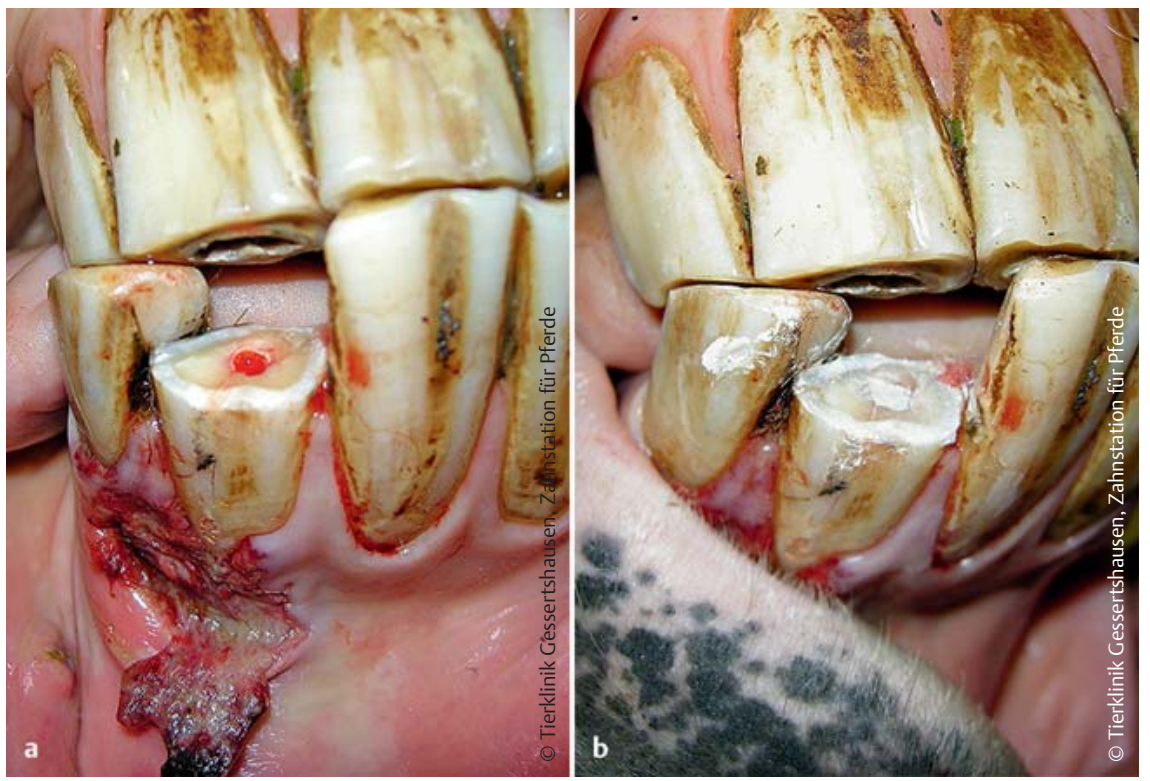

Abb. 5 a und b a Akute Schneidezahnfraktur des Zahns 402 mit Punktblutung der Pulpa und Verletzung des umliegenden Weichteilgewebes und $\mathbf{b}$ nach endodontischer Therapie.
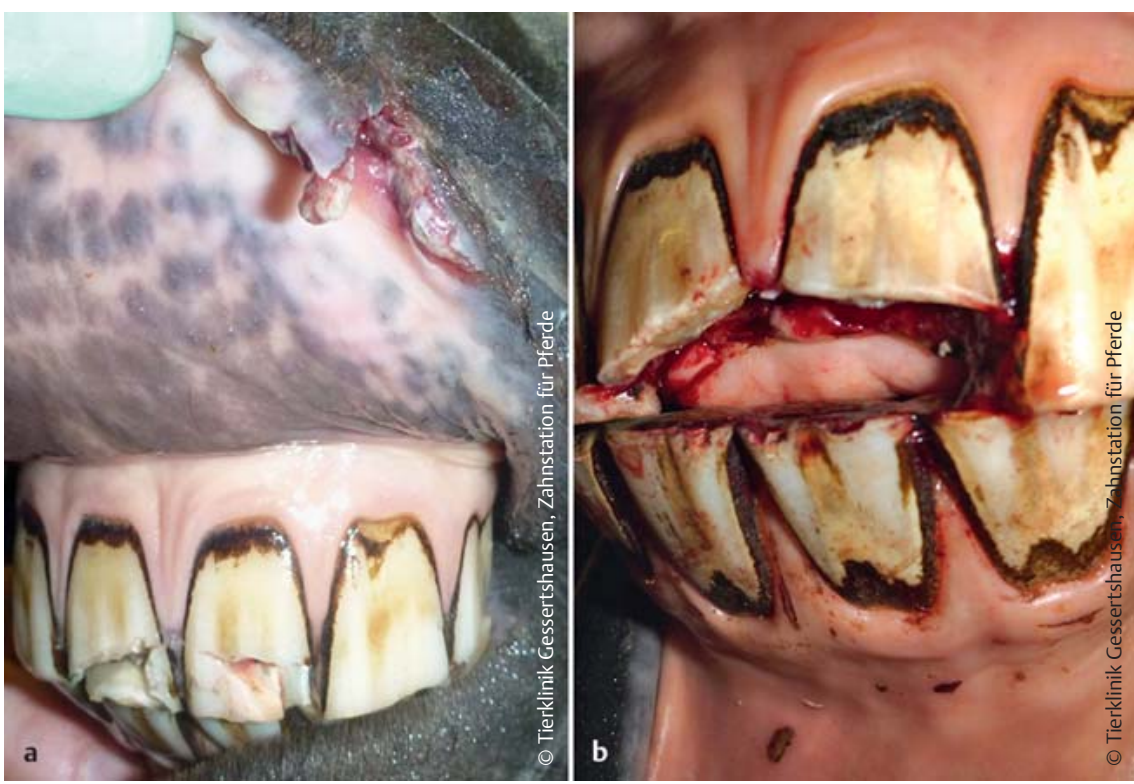

Abb. 6a und b a Akute Schneidezahnfraktur der Zähne 101 und 201 mit Verletzung der Oberlippe. b Nach Entfernung der losen Fragmente und endodontischer Therapie.

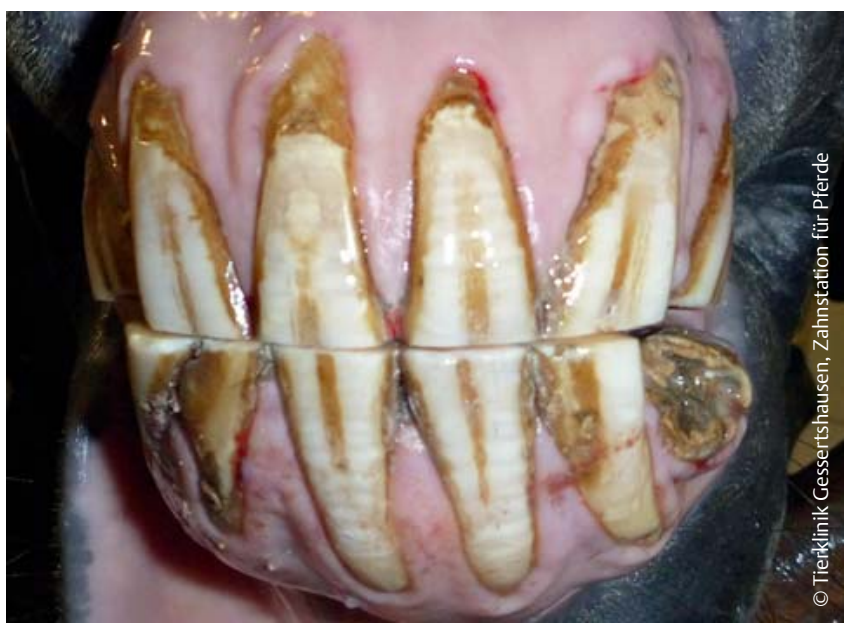

\section{Akute Schneidezahnfrakturen}

Traumatisch bedingte Frakturen der Schneidezähne gehen meist mit einer eröffneten Pulpahöhle einher und sind als zahnmedizinischer Notfall einzuordnen ( $\bullet$ Abb. 5 a und 6 a).

Können diese innerhalb von 6-12 Stunden nach dem Unfall behandelt werden, besteht die Möglichkeit der Vitalamputation, um den Zahn zu erhalten [7]. Je größer der Zeitraum zwischen Eröffnen der Pulpa und Therapie ist, desto schlechter ist die Prognose für den Erhalt der vitalen Pulpa.

Bei der Vitalamputation wird der kontaminierte Teil der Pulpa entfernt, um den Anteil der vitalen Pulpa zu erhalten. Ist die Blutung der Pulpa nicht stillbar oder die Pulpa bereits nekrotisch, eitrig oder übelriechend, wird eine Totalamputation oder Extraktion des Zahnes notwendig. Die Totalamputation kann einige Tage bis Wochen nach der Verletzung durchgeführt werden [7,9], besteht allerdings bereits eine Fistel oder chronische Wurzelspitzenentzündung, ist die Extraktion des Zahnes angezeigt.

Da eine umfangreiche Spezialausrüstung und ausreichend praktische Erfahrung zur endodontischen Therapie benötigt werden, ist in diesen Fällen die Überweisung in eine spezialisierte Klinik anzuraten.

Die Vitalamputation ( $\mathrm{Abb} . \mathbf{5 b}$ und $\mathbf{6 b}$ ) erfolgt in folgenden Schritten:

- Entfernung loser Zahnfragmente und Abrunden scharfer Kanten

- Entfernung infizierter Pulpa mit einem sterilen Bohrer

- Desinfektion mit Wasserstoffperoxid (3\%), Blutstillung

- Abdecken der Pulpa mit Kalziumhydroxid

- Verschluss mit 2 Schichten Resinzement oder einer Schicht Zement und darauf eine Kompositfüllung

- Abrunden und Polieren [7,9]

\section{Alte Schneidezahnfrakturen}

Im Praxisalltag werden häufig alte Frakturen der Schneidezähne ( $\bullet \mathbf{A b b} . \mathbf{2}$ und $\mathbf{7}$ ) entdeckt, die den Pferdebesitzern noch nicht oder erst spät aufgrund von Maulgeruch aufgefallen sind. Bei länger bestehen- 


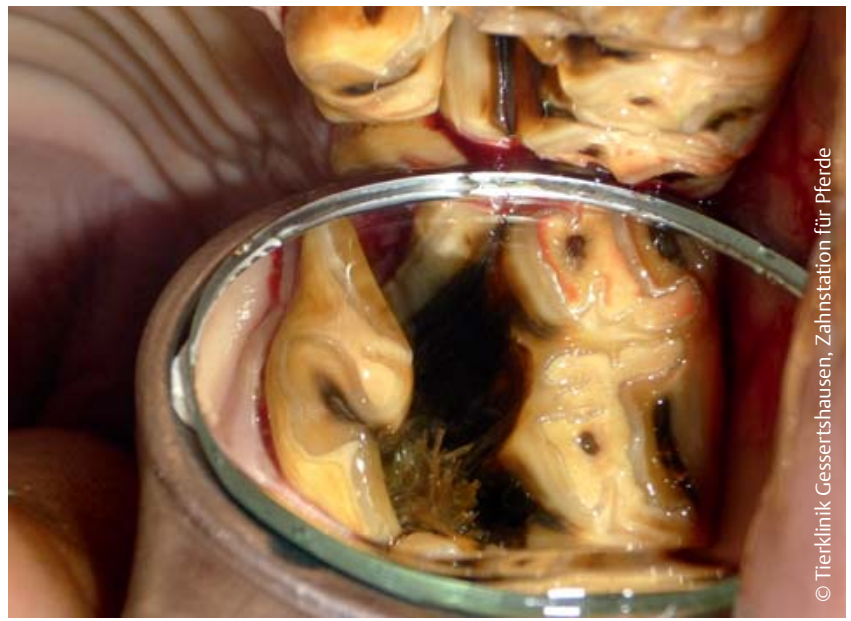

Abb. 8 Slabfraktur bzw. Sagittalfraktur durch die Pulpenpositionen 3 und 4 des Zahnes 207, impaktiertes Futter zwischen den Fragmenten.

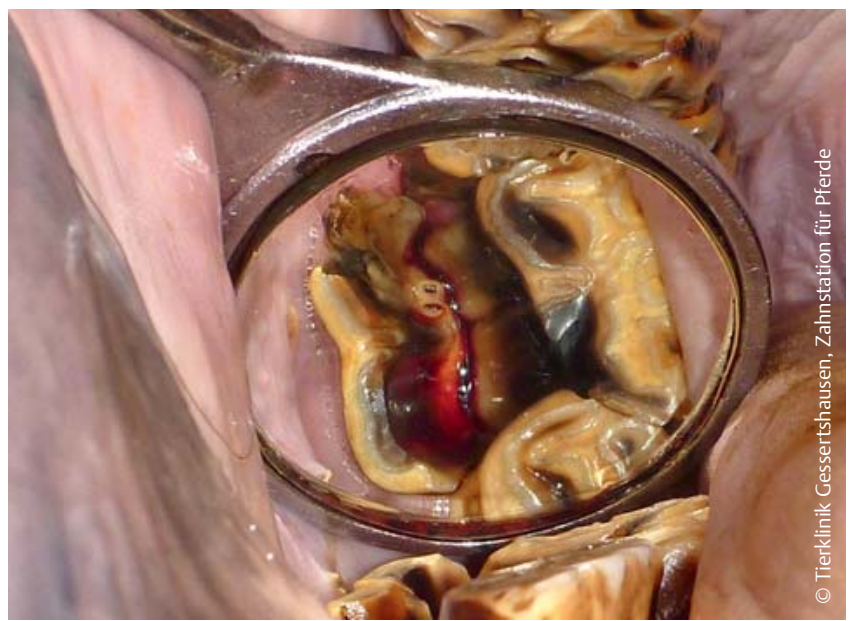

Abb. 10 Sagittalfraktur des Zahnes 407 durch die lateralen Pulpenpositionen, lockeres Fragment deutlich nach bukkal verlagert.

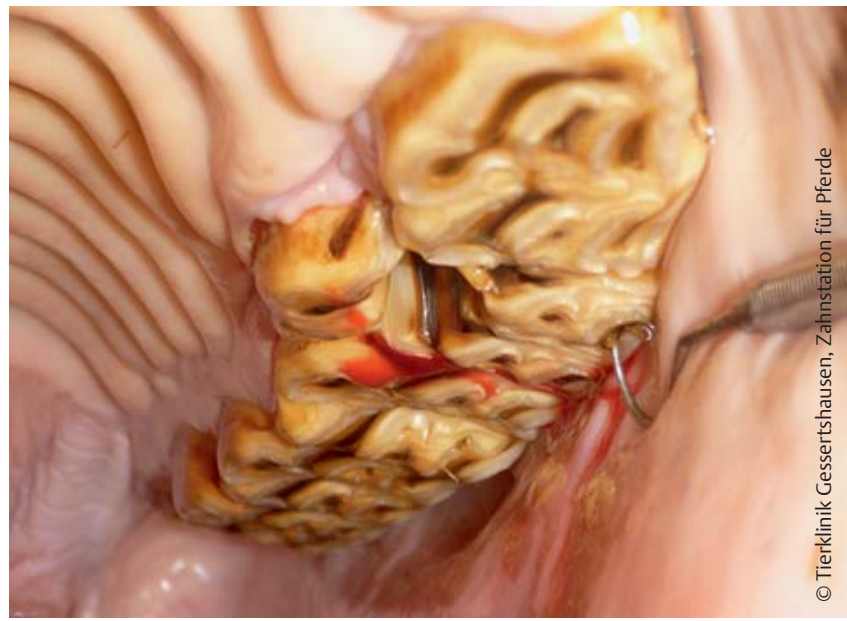

Abb.9 Sagittalfraktur 207, sondierbare Pulpa, palatinales Fragment verlagert.

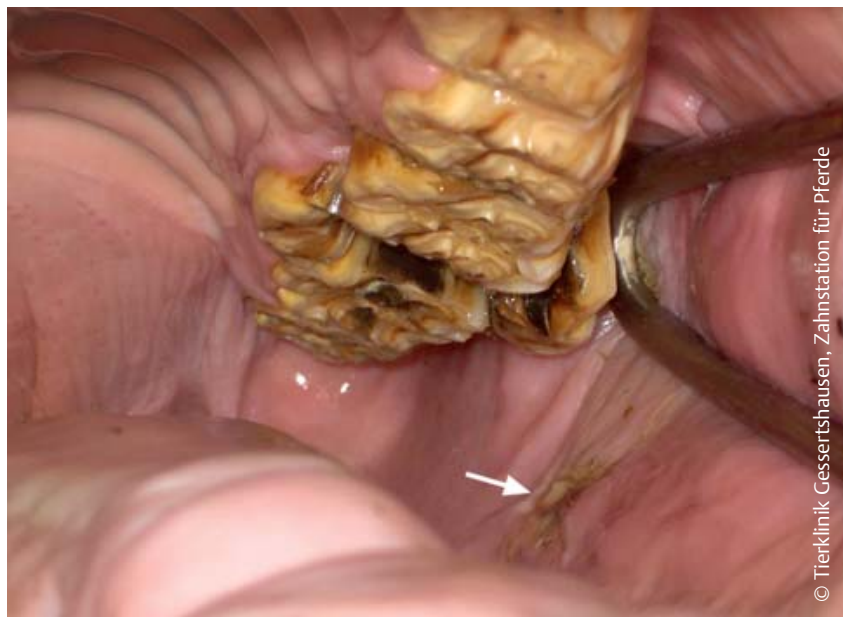

Abb. 11 Sagittalfraktur durch die Infundibula des Zahnes 209, Schleimhautulzeration bukkal (Pfeil). den Frakturen und wenn die frakturierten Zähne locker sind, ist die Prognose für den Zahnerhalt schlecht und die Zähne müssen extrahiert werden. Sollte die Schneidezahnpulpa nicht mitbeteiligt sein, genügen die Entfernung des losen Fragmentes und das Abrunden scharfer Stellen am verbleibenden Zahn [11].

Entfernung von Fragmenten und Extraktion frakturierter Schneidezähne:

- impaktiertes Futter entfernen und intensive Reinigung

- lockere Zahnfragmente mit einer Zange erfassen oder mit einem Hebel entfernen

- bei noch fest sitzenden Zahnfragmenten Gingiva mit einem Elevator labial und lingual bzw. palatinal lösen
- Vorsichtiges Spreizen im Zahnzwischenraum. Steht kein Interdentalspreizer zur Verfügung ( $\bullet$ Abb. 4), werden Elevatoren oder Hebel verwendet [9].

- Fragment gut lockern, mit einer Extraktionszange fassen und ohne großen Kraftaufwand in Richtung der Zahnachse ausziehen

- Extraktion fest in der Alveole sitzender Zahnanteile: geraden Elevator mit dem Hammer entlang der Reservekrone eintreiben und um die Zahnlängsachse drehen [11]. Gute Sicht auf den Zahnrest ist vorteilhaft. Deshalb sollten Blutungen immer wieder mit einem Mulltupfer gestillt werden und Lippen sowie Zunge von einer Hilfsperson aus dem Sichtfeld gehalten lassen werden.
- Röntgenaufnahmen zur Überprüfung auf vollständige Entfernung anfertigen

\section{Kronenteilfrakturen der Backenzähne}

Kronenteilfrakturen sind meist Absprengfrakturen, die als Zufallsbefunde auffallen. Sie werden nach ihrer Lokalisation auf der Okklusionsfläche (z.B. palatinomesial) bezeichnet ( $\mathbf{A b b} \mathbf{1}$ und 3 ). Hierbei muss die Pulpa nicht zwingend eröffnet sein, dies sollte jedoch okklusal stets mithilfe einer Sonde überprüft werden $[6,11]$, auch wenn damit nicht unbedingt eine eindeutige Aussage bezüglich des Vorliegens einer Pulpitis gemacht werden kann. Zusätzlich wird die Anfertigung von Röntgenaufnahmen empfohlen. 


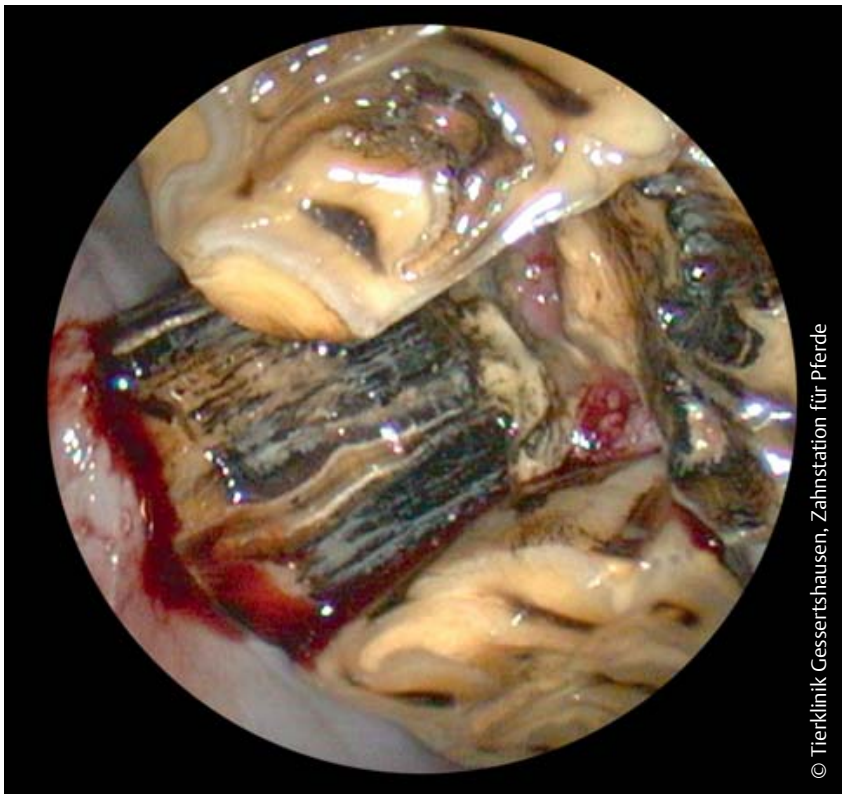

Abb. 12 Endoskopische Aufnahme einer Sagittalfraktur durch die Infundibula, die den Zahn bis in den Apikalbereich spaltet.

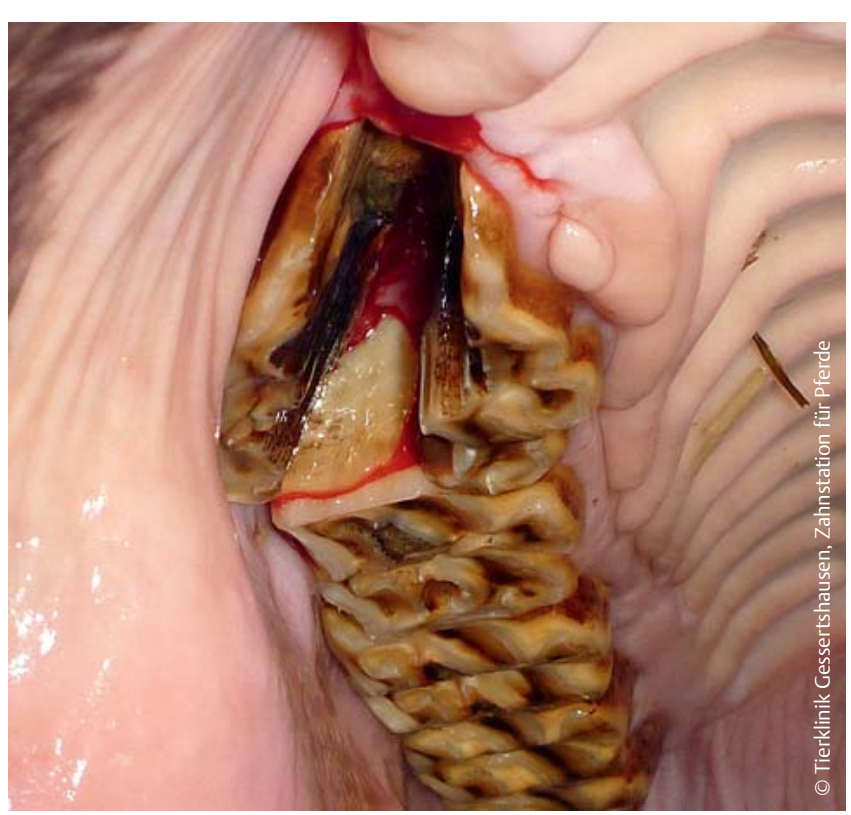

Abb. 13 Sagittalfraktur durch die kariösen Infundibula des Zahnes 106.

Häufig ist bei Kronenteilfrakturen keine Behandlung notwendig. Der aufgrund der Zahnfraktur entstandene Defekt kann durch die Eruption und die physiologische Abnutzung der Zähne allmählich verschwinden [6]. Wichtig sind eine lückenlose Dokumentation und Nachkontrollen im Rahmen der Routinezahnbehandlung alle 6-12 Monate.

\section{Sagittalfrakturen mit Beteiligung der Pulpenpositionen (Slabfrakturen)}

Diese Form von Backenzahnfrakturen ist vielfach Anlass für einen Anruf im Notdienst. Aufgrund loser und verlagerter Zahnfragmente, die Zunge oder Wange verletzen können oder der Ansammlung von Futter zwischen den Zahnfragmenten, kommt es zu akuten klinischen Symptomen ( $\triangleright$ Abb. 8, 9 und 10).

In den meisten Fällen ist eine Entfernung des losen Fragmentes als Behandlung ausreichend, wenn keine Apikalinfektion besteht [4]:

- impaktiertes Futter mit Picks oder Wasserspülung entfernen

- das lockere Fragment mit Elevatoren von der Gingiva lösen

- Ansetzen eines Picks im Frakturspalt. Hebeln nach bukkal oder lingual/palatinal und den losen Chip mit einer Zange erfassen 


\section{ps.fachspiegel}

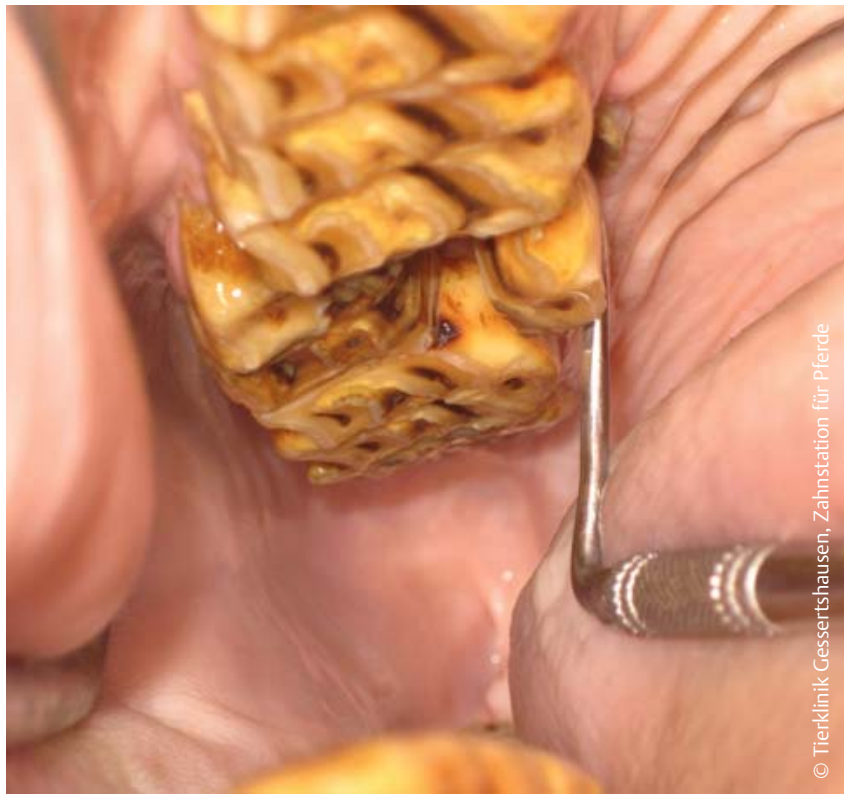

Abb. 14 Die Gingiva wird palatinal mittels Elevator von dem losen Zahnfragment gelöst.

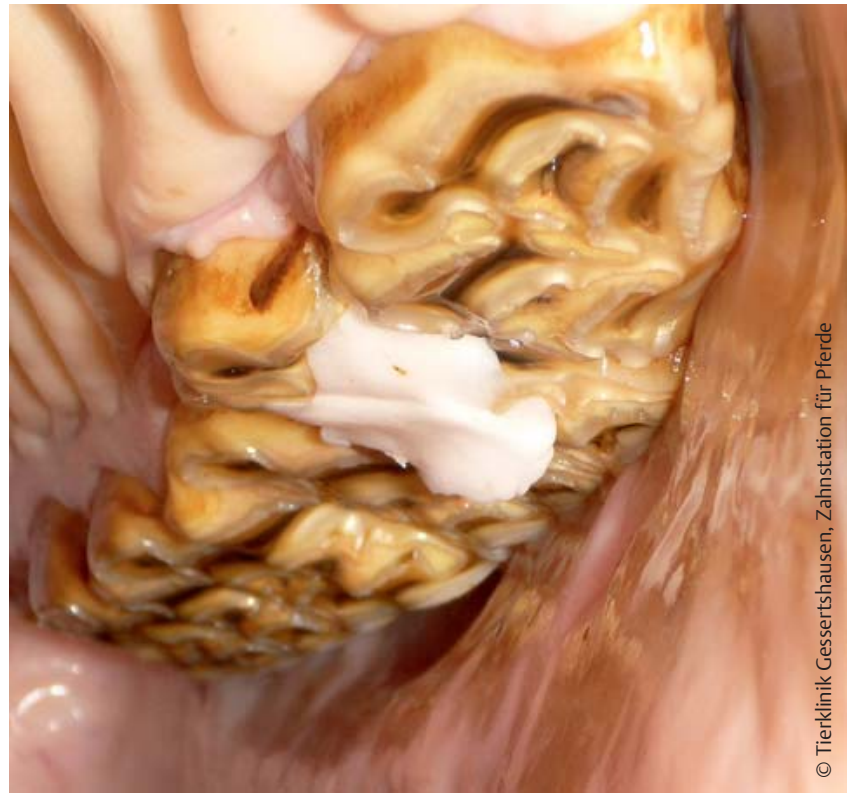

Abb. 15 Frakturierter Zahn nach Auffüllen des Defektes mit Demotec ${ }^{\circledR}$.
- Abrunden scharfkantiger Stellen am verbleibenden Zahn und Gegenspieler aus der Okklusion nehmen

- gegebenenfalls Röntgenaufnahmen nach der Entfernung des Fragmentes anfertigen

Ziel der Behandlung ohne Extraktion des Zahnes ist die Regeneration der Pulpenpositionen durch Bildung von Tertiärdentin und die Wiederherstellung der Okklusionsfläche durch Nachschieben des Zahnes [4]. Verlaufskontrollen unter Umständen mit Kontrollröntgenaufnahmen im Abstand von 6 Monaten sind empfehlenswert. Bei akuten Zahnfrakturen liegt meist kein Hinweis auf eine Apikalinfektion vor, es können im weiteren Verlauf allerdings Komplikationen auftreten.

Bei klinischen und radiologischen Anzeichen für eine Apikalinfektion wird eine Extraktion des Zahnes notwendig [4]. Zur Apikalinfektion scheint es in $24 \%$ dieser Fälle zu kommen [3]. Nach der Entfernung des Fragments kann meist erst durch den freien Blick auf die Frakturlinie eine mögliche Pulpenbeteiligung beurteilt werden. Liegt die Fraktur unterhalb des Zahnfleischniveaus, können tiefe Parodontaltaschen entstehen [11].

\section{Sagittalfrakturen durch die Infundibula der Backenzähne}

Durch Infundibularkaries der Oberkieferbackenzähne kann es zum Substanzverlust und zur Fraktur betroffener Zähne kommen ( $\triangleright$ Abb. 11). Aufgrund der meist vollständigen Spaltung des Zahnes bis in den Apikalbereich ( $\bullet$ Abb. 12 und 13) wird eine Extraktion notwendig [10]. Diese Form der Backenzahnfrakturen führt zu $100 \%$ zur Apikalinfektion des betroffenen Zahnes [3].

Sollten noch beide Zahnteile vorhanden sein, kann die Extraktion durch Verkleben der Anteile bzw. temporäres Füllen des Zahndefektes erleichtert werden, da hiermit die Stabilität des Zahnes erhöht wird [10].

Hierbei geht man wie folgt vor:

- Kürzen des Gegenspielers

- intensive Reinigung des Frakturspaltes mit Hilfe von Picks und Wasserspülung

- Gingiva mit Elevatoren von den Fragmenten lösen ( $\bullet$ Abb. 14). Bei sehr lockeren Fragmenten erfolgt dieser Schritt nach der Füllung des Zahndefektes.

- Zusammenpressen der beiden Anteile mit einer Extraktionszange

- Auffüllen des Defektes z.B. mit Kunstharz (Demotec $\left.{ }^{\circledR}\right)(\triangleright$ Abb. 15) und unter Zusammenpressen der Fragmente aus-

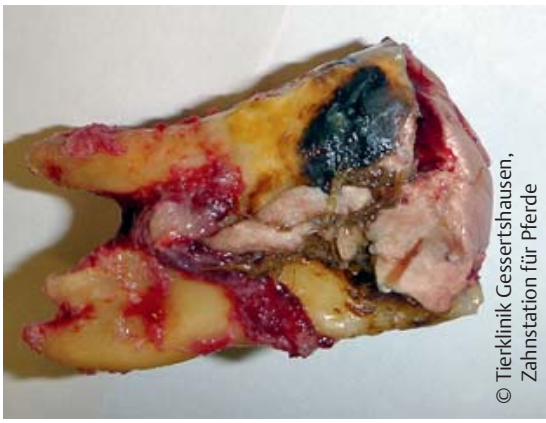

Abb. 16 Temporär gefüllter Backenzahn nach Extraktion.

härten lassen. Währenddessen Überstände wegstreichen und nach Möglichkeit mit Wasser kühlen, da das Füllmaterial warm wird.

- Es folgt die Extraktion des Zahnes in den bekannten Schritten: Spreizen, Lockern, Extraktion mithilfe eines Hypomochlions ( Abb. 16) [10].

Der zu extrahierende Zahn kann, je nachdem wie lange der Krankheitsprozess bereits besteht, zwischen den Nachbarzähnen eingekeilt sein, da das Auseinanderweichen der Fragmente ein Annähern der umliegenden Zähne bedingt. Dieser Zustand kann die Extraktion erschweren, der Zahn muss beim Auszug nach palatinal abgekippt werden oder die Nachbarzähne müssen mit einer Fräse bearbeitet werden, um Platz zu schaffen. Hierbei sind 
in jedem Fall die Pulpen der gesunden Zähne zu schonen.

Fehlt bereits ein Zahnanteil oder frakturiert der Zahn während der Extraktion weiter, kann sich die Extraktion komplizierter gestalten. Hier muss auf weiterführende Techniken der Zahnentfernung (Bukkotomie, intraorale Segmentation) zurückgegriffen werden [10].

\section{Fazit}

Der Zahnerhalt steht an erster Stelle, kann aber bei Zahnfrakturen nicht immer gewährleistet werden. Eine endodontische Therapie zum Erhalt frakturierter Schneidezähne ist dann sinnvoll, wenn das Trauma zeitig erkannt wird. In allen weiteren Fällen muss abgewogen werden, ob eine Totalamputation sinnvoll ist oder der Zahn extrahiert werden muss.

Bei Schneidezahnfrakturen mit kleinen abgesplitterten Fragmenten und bei Sagittalfrakturen der Backenzähne mit Beteiligung der Pulpenpositionen ist in der Mehrzahl der Fälle eine Entfernung der losen Zahnanteile und Abrundung scharfer Stellen am Zahn ausreichend. Dieses Vorgehen ist nur dann empfehlenswert, solange keine Öffnung der Pulpa vorliegt.

Manche Frakturen, wie z.B. Sagittalfrakturen durch die Infundibula, erfordern nahezu immer eine Zahnextraktion. Der betroffene Zahn sollte aufgrund der Gefahr einer Apikalinfektion möglichst zeitnah entfernt werden. Fehlen große Anteile eines Zahnes, muss stets bedacht werden, dass der Zahn von den Nachbarzähnen im Laufe der Zeit eingeengt wird und die Extraktion daher nicht lange aufgeschoben werden kann. Wichtig ist eine gewissenhafte Aufklärung der Besitzer, da auch bei zunächst unkompliziert erscheinenden Zahnfrakturen im weiteren Verlauf Komplikationen auftreten können, die gegebenenfalls zum Zahnverlust führen, wie z. B. bei Anzeichen einer Apikalinfektion oder Pulpitis. Regelmäßige Kontrollen werden empfohlen.
1 Baker GJ. Verletzungen der Zähne. In: Baker G], Easley J (Hrsg.): Zahnheilkunde in der Pferdepraxis. München: Elsevier; 2007: 93-96

2 Dacre I, Kempson S, Dixon PM. Equine idiopathic cheek teeth fractures. Part 1: Pathological studies on 35 fractured cheek teeth. Equine Vet J 2007; 39(4): 310-318

3 Dacre IT. Pathologie. In: Baker G], Easley J, Hrsg. Zahnheilkunde in der Pferdepraxis. München: Elsevier; 2007: 111-114

4 Dixon PM, Barakzai SZ, Collins NM et al. Equine idiopathic cheek teeth fractures: Part 3: A hospital-based survey of 68 referred horses (19992005). Equine Vet J 2007; 39(4): 327-332

5 Dixon PM. Idiopathic Cheek Teeth Fractures. IGFP Proceedings, 2012: 47-49

6 Simhofer $H$, Zetner $K$. Krankheiten der Mundhöhle, der Zähne, der Zunge und der Kiefer. In: Dietz O, Huskamp B, Hrsg. Handbuch Pferdepraxis. Stuttgart: Enke; 2006: 389-390

7 Stoll M. Endodontische Therapien an den Schneidezähnen des Pferdes. Pferdespiegel 2011; 1: 24-30

8 Taylor L, Dixon PM. Equine idiopathic cheek teeth fractures: Part 2: A practice- based survey of 147 affected horses in Britain and Ireland. Equine Vet J 2007; 39(4): 322-326

9 Vogt C, Bienert-Zeit A, Simhofer $H$ et al. Schneidezahnerkrankungen. CVE 2016; 4(4): 1-32

10 Zwick T. Die orale Extraktion sagittal frakturierter Backenzähne. IGFP Proceedings, 2013: 172 177

11 Zwick T. Zahnfrakturen. In: Vogt, C, Hrsg. Lehrbuch der Zahnheilkunde beim Pferd. Stuttgart: Schattauer. 2011; 160-164

\section{Online}

https://doi.org/10.1055/s-0043-119164

\section{Dr. Anna Altersberger}

Zahnstation der Tierärztlichen Klinik Gessertshausen

Grasweg 2

86459 Gessertshausen

www.zahnstation-gessertshausen.de anna.altersberger@gmx.de 